\title{
Parameter study of self-absorption effects in Total Reflection X-ray Fluorescence-X-ray Absorption Near Edge Structure analysis of arsenic ${ }^{\text {tr }}$
}

\author{
F. Meirer ${ }^{\text {a, } *}$, G. Pepponi ${ }^{\text {b }}$, C. Streli ${ }^{\text {a }}$, P. Wobrauschek ${ }^{\text {a }}$, P. Kregsamer ${ }^{\text {a }}$, N. Zoeger ${ }^{\text {a }}$, G. Falkenberg ${ }^{\text {c }}$ \\ a Atominstitut, Vienna University of Technology, 1020 Wien, Austria \\ b Fondazione Bruno Kessler (FBK), via Sommarive 18, 38100 Povo (Trento), Italy \\ c Hamburger Synchrotronstrahlungslabor at DESY, 22607 Hamburg, Germany
}

\section{A R T I C L E I N F O}

\section{Article history:}

Received 31 October 2007

Accepted 14 May 2008

Available online 29 May 2008

\section{Keywords:}

TXRF

XANES

Self-absorption

Arsenic

\begin{abstract}
A B S T R A C T
Total reflection X-ray Fluorescence (TXRF) analysis in combination with X-ray Absorption Near Edge Structure (XANES) analysis is a powerful method to perform chemical speciation studies at trace element levels. However, when measuring samples with higher concentrations and in particular standards, damping of the oscillations is observed. In this study the influence of self-absorption effects on TXRF-XANES measurements was investigated by comparing measurements with theoretical calculations. $\mathrm{As}(\mathrm{V})$ standard solutions were prepared at various concentrations and dried on flat substrates. The measurements showed a correlation between the damping of the oscillations and the As mass deposited. A Monte-Carlo simulation was developed using data of the samples shapes obtained from confocal white light microscopy. The results showed good agreement with the measurements; they confirmed that the key parameters are the density of the investigated atom in the dried residues and the shape of the residue, parameters that combined define the total mass crossed by a certain portion of the incident beam. The study presents a simple approach for an a priori evaluation of the self-absorption in TXRF X-ray absorption studies. The consequences for Extended $\mathrm{X}$-ray Absorption Fine Structure (EXAFS) and XANES measurements under grazing incidence conditions are discussed, leading to the conclusion that the damping of the oscillations seems to make EXAFS of concentrated samples non feasible. For XANES "fingerprint" analysis samples should be prepared with a deposited mass and sample shape leading to an acceptable absorption for the actual investigation.
\end{abstract}

(c) 2008 Elsevier B.V. All rights reserved.

\section{Introduction}

Total Reflection X-ray Fluorescence Analysis (TXRF) is a sensitive technique for qualitative and quantitative elemental determination of traces. Typical TXRF set-ups use multilayer monochromators as preferred compromise among high primary flux and low spectral background $[1,2]$. Synchrotron radiation TXRF offers detection limits in the fg range for transition metals with a multilayer monochromator and a bending magnet beamline $[3,4]$.

For dilute samples X-ray Absorption Spectroscopy (XAS) is commonly performed in fluorescence mode as the fluorescence signal is proportional to the absorption coefficient and it gives a better signal to background ratio. With a perfect crystal monochromator TXRF acquisition can be utilized for XAS to gain chemical information on the element of interest. With this modified set-up there is a flux reduction of about two orders of magnitude, but it is still sufficient for the

\footnotetext{
it This paper was presented at the 12th Conference on Total Reflection X-ray Fluorescence Analysis and Related Methods held in Trento (Italy), 18-22 June 2007, and is published in the Special Issue of Spectrochimica Acta Part B, dedicated to that conference.

* Corresponding author. Atominstitut, Vienna University of Technology, Stadionallee 2, 1020 Wien, Austria. Tel.: +4315880154 155; fax: +4315880114199.

E-mail address: fmeirer@ati.ac.at (F. Meirer).
}

analysis at ppb level [3,5-7]. This approach allows the extension of XAS to traces in droplet samples where only small amounts are available. Such measurements can also be performed for the speciation of light elements using a plain grating monochromator [8].

Fluorescence acquisition of XAS spectra of concentrated sample suffers from self-absorption which causes damping and also broadening of the oscillations. Some authors have performed quantitative speciation through analysis of XANES (X-ray Absorption Near Edge Structure) spectra by fitting them with analytical functions $[9,10]$.

Another approach deals with the corrections of the measured spectra to account for self-absorption. Many authors have investigated self-absorption effects in XAS using fluorescence acquisition depending on the angle of incidence and detection and have proposed correction models [11,12]. Due to irregular sample shape and the very shallow angle of incidence these models are not applicable to TXRF. The extreme grazing incidence geometry used by TXRF enhances these self-absorption effects due to the extended path length of the incident beam in the droplet. This path length is equivalent to the penetration depth of the incident beam and is therefore energy dependent. As the energy changes during a XANES scan the size of volume where the fluorescence photons originate from is varying. Higher absorption means smaller excited volume and therefore less fluorescence intensity (and vice versa). Consequently this leads to a 
damping of the oscillations of the absorption coefficient. The phenomenon is relevant at higher concentrations, such as would be used in the measurements of standards to have a good counting statistic in relatively short measurement time. Any absorption of the fluorescence radiation within the sample can be neglected because it remains constant during an energy scan.

In general TXRF is known to allow for linear calibration typically using an internal standard for quantification [1,2]. In fact for the fluorescence radiation collected by the detector the sample is "thin" and differential absorption for photons with different energies can be ignored. Assuming the sample to be homogeneously distributed the loss of fluorescence signal due to absorption of the primary beam equally affects all elements and quantification by using an internal standard is not affected by the phenomenon.

Preliminary investigations showed a significant damping of the XANES spectra of standard samples [6,7]. This effect could be correlated to self-absorption effects. It is therefore of interest to investigate the absorption effect depending on the total mass deposited. The three-dimensional shape of the sample and the density of the element investigated are key parameters.

\section{Experimental}

Two series of samples have been measured. The first series consist of three samples on Plexiglas reflectors. Different total amounts of masses (1,10 and $100 \mathrm{ng}$ ) of arsenic were applied onto the reflectors. The second series contained samples on quartz reflectors with masses of $4,9,20,72,100$ ng of arsenic and, additionally, one sample on a Plexiglas carrier containing $500 \mathrm{ng}$. This second series was analyzed with a confocal optical microscope to gain information about the 3-dimensional shape of the dried residues. For both series arsenic K-edge XANES measurements in fluorescence mode and grazing incidence geometry were performed. The results of these measurements were compared with the output of a simple Monte-Carlo simulation of absorption effects performed for each sample.

\subsection{Sample preparation}

A set of arsenic containing solutions had been prepared from a $1000+/-5 \mathrm{mg} / \mathrm{L}$ solution (MERCK CertiPUR, traceable to SRM from NIST, $\mathrm{H}_{3} \mathrm{AsO}_{4}$ in $\mathrm{HNO}_{3}$, \#1.19773.0100). Tri-distilled water (Atominstitut) was used for the dilution of the mother solution to obtain the following set of As concentrations: 1, 4, 10, 20, 40, 80, 100, 300, $500 \mathrm{mg} / \mathrm{L}$. The respective volumes of mother solution and tri-distilled water were pipetted into a SARSTEDT flask, but the actual dilution factors were gained by the readings of a suited balance (SARTORIUS R300S)

$1 \mu \mathrm{L}$ of each of these solutions was pipetted onto a quartz or Plexiglas reflector (both $30 \mathrm{~mm}$ diameter) respectively. Prior to the pipetting a blank measurement was done for each of these reflectors to assure that no $\mathrm{As}, \mathrm{Pb}$, etc. contamination would falsify the results. These measurements were performed with an ATOMIKA Extra II (LT: $100 \mathrm{~s}, 50 \mathrm{kV}, 38 \mathrm{~mA}$ ). The beam spot size and the area inspected by the detector of the EXTRA II were in the range of various millimeters, therefore the examined area was much larger than the pipetted samples. The pipette (EPPENDORF research $0.1-2.5 \mu \mathrm{L}$ ) showed for this volume a maximum error of $+/-20 \%$, consequently the pipetting step served only for sample positioning in the centre of the respective reflectors and the mass was determined by differential weighing:

Series 1 (Plexiglas refl.): 1.10, 10.02, $99.8 \mathrm{ng}$

Series 2 (quartz, refl.): 3.98, 8.99, 20.0, 71.6, $100.2 \mathrm{ng} \& 503.5 \mathrm{ng}$ (Plexiglas refl.)

The aqueous/acidic matrix was removed by drying the reflectors on a hot plate inside a flow hood in order to avoid contaminations.

\subsection{TXRF-XANES measurements}

Arsenic K-Edge XANES measurements in fluorescence mode and grazing incidence geometry were carried out using the set-up at the beamline $L$ at the Hamburger Synchrotronstrahlungslabor (HASYLAB) at DESY $[3,4]$. All measurements were performed in vacuum. A Si(111) double crystal monochromator was used for selecting the energy of the exciting beam from the continuous X-ray spectrum emitted by the 1.2 Tesla bending magnet at beamline $\mathrm{L}$. The primary beam was collimated to $200 \mu \mathrm{m} \times 2000 \mu \mathrm{m}$ (horizontal ×vertical) by a cross-slit system. The incident X-ray intensity was monitored with an ionization chamber.

During the measurements the excitation energy was tuned in varying steps ( $5 \mathrm{eV}$ to $0.5 \mathrm{eV}$ ) across the arsenic K-edge at $11,867 \mathrm{eV}$. At each energy a fluorescence spectrum was recorded by a Silicon Drift Detector (SDD, VORTEX $50 \mathrm{~mm}^{2}$, Radiant Detector Technologies) $[13,14]$. The distance between SDD and sample carrier was $1 \mathrm{~mm}[3]$. For series 1 (Plexiglas reflectors) the acquisition time for each spectrum was set to $10 \mathrm{~s}$ for the $1 \mathrm{ng}$ sample and to $4 \mathrm{~s}$ for the 10 and $100 \mathrm{ng}$ sample. Each scan consisted of 305 spectra and the energy range was set from 11,700 to $12,300 \mathrm{eV}$. For series 2 (quartz reflectors) the acquisition times were set to $1 \mathrm{~s}$ for the samples containing masses of 300 and $500 \mathrm{ng}$ and to $2 \mathrm{~s}$ for the rest of the samples. The energy range was set from 11,700 to $12,500 \mathrm{eV}$ and 576 spectra were recorded for each scan of this series. Simultaneously, the absorption by an elemental gold foil was recorded in transmission mode for each scan of both series. The first inflection point (i.e. the first maximum of the derivative spectrum) of the Au metal foil scan was assumed to be 11,919 eV (Au-L3 edge). As described the data acquisition time was increased for samples with lower total amounts of arsenic. This was done to achieve better statistics for the evaluation of the As fluorescence peak area. From these peak areas the XANES spectra were built and therefore a specific minimum count rate was desirable.

As the critical angle of total reflection changes slightly $(<0.2 \mathrm{mrad}$ for silicon) during the energy scan the incident angle of the primary X-ray beam was adjusted to $2 \mathrm{mrad}$, which is far below the critical angle $(\sim 2.7 \mathrm{mrad}$ at $11,700 \mathrm{eV})$. On that account it was assumed that the change of the critical angle during the XANES scans was unproblematic for the measurement of droplet samples (residues on surface).

Both measured and simulated absorption spectra have been analyzed with ATHENA which is included in the IFEFFIT program package for XAS analysis [15-17]. Using this software each scan was normalized and its energy scale was corrected with respect to the AuL3 edge. Multiple scans of the same sample have been merged by calculating the average and standard deviation at each point in the set.

\subsection{Confocal microscopy}

Measurements to determine the shape of the samples of series 2 (quartz reflectors) have been performed utilizing a confocal white light microscope (NanoFocus $\mu$ surf® [18]). The analyses were done by the Austrian Center of Competence for Tribolgy (AC2T). The measuring field was $\sim 1450 \times 1400 \mu \mathrm{m}$ with a lateral resolution of $\sim 1.5 \times 1.5 \mu \mathrm{m}$ and $50 \mathrm{~nm}$ in height. Due to the measurement set-up it was necessary to perform a plane correction and flattening of the data.

The raw confocal microscope images were first leveled using a polynomial fit of grade 1, then the zero point of the $z$ axis was set to the maximum of the histogram of the heights. The procedure was carried out using the software package SPIP 4.2.6.0 [19]. Additionally the data was treated using a threshold filter. This was done mainly because the amount of data had to be reduced to obtain reasonable simulation times. The threshold filter removed each data point with a height smaller than $25 \%$ of the maximum height of the sample. The result was a simplified sample with respect to the real sample's shape-speckles of questionable origin (traces of the sample or measurement artifacts) which were found on the reflectors surface have been removed. 
The result of measurements and data treatment was a matrix representing the three-dimensional distribution of the sample on the reflector's surface (see Fig. 2). This information was used for the simulation of absorption effects during a TXRF-XANES scan considering the sample's shape (see Section2.4).

\subsection{Development of a simple Monte-Carlo simulation}

To simulate the observed damping of the XANES oscillations (see Fig. 4) for samples with higher mass a simple Monte-Carlo simulation was developed.

XAS data obtained for the sample with the smallest mass was taken as reference XANES scan for the Monte-Carlo simulation (i.e. $1 \mathrm{ng}$ for the samples on Plexiglas reflectors (series 1 ) and $4 \mathrm{ng}$ for the samples on quartz reflectors (series 2)). On the basis of preliminary investigations [6,7] which showed damping effects for higher masses (>10 ng) it was assumed that these scans show no significant damping of the oscillations of the fine structure. Each reference scan was normalized within the ATHENA software package by edge step normalization [17]. With this procedure the pre-edge region of the scan was normalized to zero while the post-edge region was normalized to one. The total absorption cross section $\mu$ used for the simulation has been calculated from the tabulated values for the photoelectric absorption cross section $\tau$ published by Henke et al. [20] and the scattering cross sections $\left(\sigma_{\text {coh }}\right.$ and $\left.\sigma_{\text {incoh }}\right)$ published by Ebel et al. [21]:

$\mu=\tau+\sigma_{\text {coh }}+\sigma_{\text {incoh }}$

For the calculation the value of the density of As in the actual sample is required (this is influenced by hydration when crystallizing in the process of drying and the presence of other elements present in the solution). Thus the $\mu$ used is linked to the coefficient for elemental arsenic through the following relationship:

$\mu=\mu_{\text {elemental }}(E) \times\left(B / \rho_{\text {As_elemental }}\right)$

$\rho_{\text {As_elemental }}=5.73 \mathrm{~g} / \mathrm{cm}^{3}$ (as tabulated for elemental arsenic)

B ("corrected density") is the actual density of the As in the sample

Here it was assumed that all the absorption in the sample is given by the As (the standard used is $\mathrm{H}_{3} \mathrm{AsO}_{4}$ in $\mathrm{HNO}_{3}$, and there might be some hydration in the crystallization, but there are no heavy, strongly absorbing elements-this was verified by checking the single spectra of a scan for contaminations).

The energy scale of the measured data was corrected by shifting the maximum of the first derivative of the XANES scan to the maximum of the first derivative of the theoretical $\tau$ function. The Kedge contribution $\tau_{\mathrm{K}}$ to the total photoabsorption coefficient $\tau$ can be calculated according to

$\tau_{\mathrm{K}}(E)=\tau(E) \times\left(S_{\mathrm{K}}-1\right) /\left(S_{\mathrm{K}}\right)$

where $S_{\mathrm{K}}$ is the edge jump ratio.

For the simulation the tabulated absorption coefficients of a free atom were modified by superimposing the fine structure of the absorption coefficient measured in the reference spectrum. First a modified $\tau_{\mathrm{K}}$ coefficient was calculated as follows:

$\tau_{\mathrm{K}, \text { calc }}(E)=\left\{\begin{array}{cc}f_{\mathrm{ex}}(E) \cdot S_{\mathrm{K}} & \ldots E<\text { absorption edge } \\ \tau_{\mathrm{K}}(E) \cdot f_{\mathrm{ex}}(E) & \ldots E>\text { absorption edge }\end{array}\right.$

where $f_{\text {ex }}(E)$ represents the normalized values of the reference scan.

In the next step the tabulated values of $\tau$ have been modified as follows:

$\tau_{\text {calc }}(E)=\left\{\begin{array}{cc}\tau(E)+\tau_{\mathrm{K}, \text { calc }}(E) & \ldots E<\text { absorption edge } \\ \tau(E)-\tau_{\mathrm{K}}(E)+\tau_{\mathrm{K}, \text { calc }}(E) & \ldots E>\text { absorption edge }\end{array}\right.$
The total absorption cross section $\mu_{\text {calc }}(E)$ was determined according to Eq. (1) and furthermore used for all calculations within the Monte-Carlo simulation.

Starting from a uniformly distributed random number $P$, interpreted as absorption probability of a photon in the sample, an absorption length $L_{\mathrm{abs}}$ was generated utilizing Beer-Lambert's Law:

$L_{\mathrm{abs}}(E)=-\log _{e}(P) / \mu_{\mathrm{calc}}(E)$

where $P(=I / I 0) \in] 0,1]$

The absorption length is the path length of a photon propagating in the sample until it gets absorbed. For each incident photon penetrating the sample, the length $L_{\mathrm{inc}}$ of its path through the sample was compared with its absorption length $L_{\mathrm{abs}}$. If the absorption length was found to be smaller than the incident photon's path length $L_{\text {inc }}$ $\left(L_{\mathrm{abs}}<L_{\mathrm{inc}}\right)$ an arsenic K-alpha fluorescence photon was counted with the probability $\tau_{\mathrm{K} \text {,calc }}(E) / \mu_{\text {calc. }}$. To simulate a whole XANES scan a fixed number of incident photons was used for each energy. The simulated XANES spectra were built from the counted K-alpha fluorescence photons per energy value generated as described above. The software for the simulation was written in $\mathrm{C}++$ using the TT800 random generator [22] with a period of $2^{\wedge} 800$ to generate the random number $P$. The other input parameters $L_{\mathrm{inc}}$ and $B$ have been determined in different ways for the two series of samples.

For the samples which were investigated with the confocal microscope (series 2) the real sample's dimensions were known from these measurements. Therefore the volume of each sample could be easily calculated to determine the density $B$ :

$B=m_{\mathrm{S}} / V_{\mathrm{c}}$

$V_{\mathrm{c}}$ : volume of the sample

$m_{\mathrm{s}}$ : Arsenic mass

For these samples not only one $L_{\text {inc }}$ value was calculated but a set of paths with different lengths depending on the shape of the sample. Assuming a non-divergent beam parallel to the reflectors' surface, the length of each path became a function of the $Y$ and $Z$ coordinate of the origin of the exciting photon (i.e. position where the incident photon entered the sample, see Fig. 1).

The set of paths for each sample was calculated with respect to the lateral resolution of the confocal microscope $(\sim 1.5 \mu \mathrm{m} \times 1.5 \mu \mathrm{m})$.
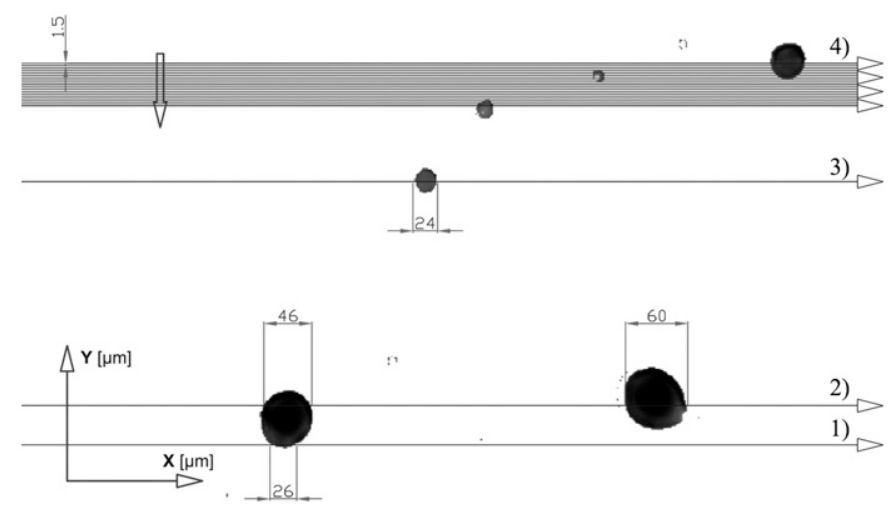

Fig. 1. One zoomed region of the " $72 \mathrm{ng} \mathrm{As}(\mathrm{V})$ " sample of series 2 . The dark spots are some of the dried residues of the pipetted sample. The arrows labeled 1-3 show three different possible paths of a photon propagating parallel to the sample carrier. It can be seen that for each of these three paths the photon covers different distances in the dried residues. These different distances (e.g. $106 \mu \mathrm{m}$ for path 2) are indicated in microns. To get a set of distances for all possible paths through the sample a path was calculated every $1.5 \mu \mathrm{m}$ (lateral resolution of the confocal microscope) along the $Y$-axis. This is indicated in the upper part of the figure (label 4). The calculation of the distances produced by each path was done for the entire $Y$-range of the measuring field of the confocal microscope. 


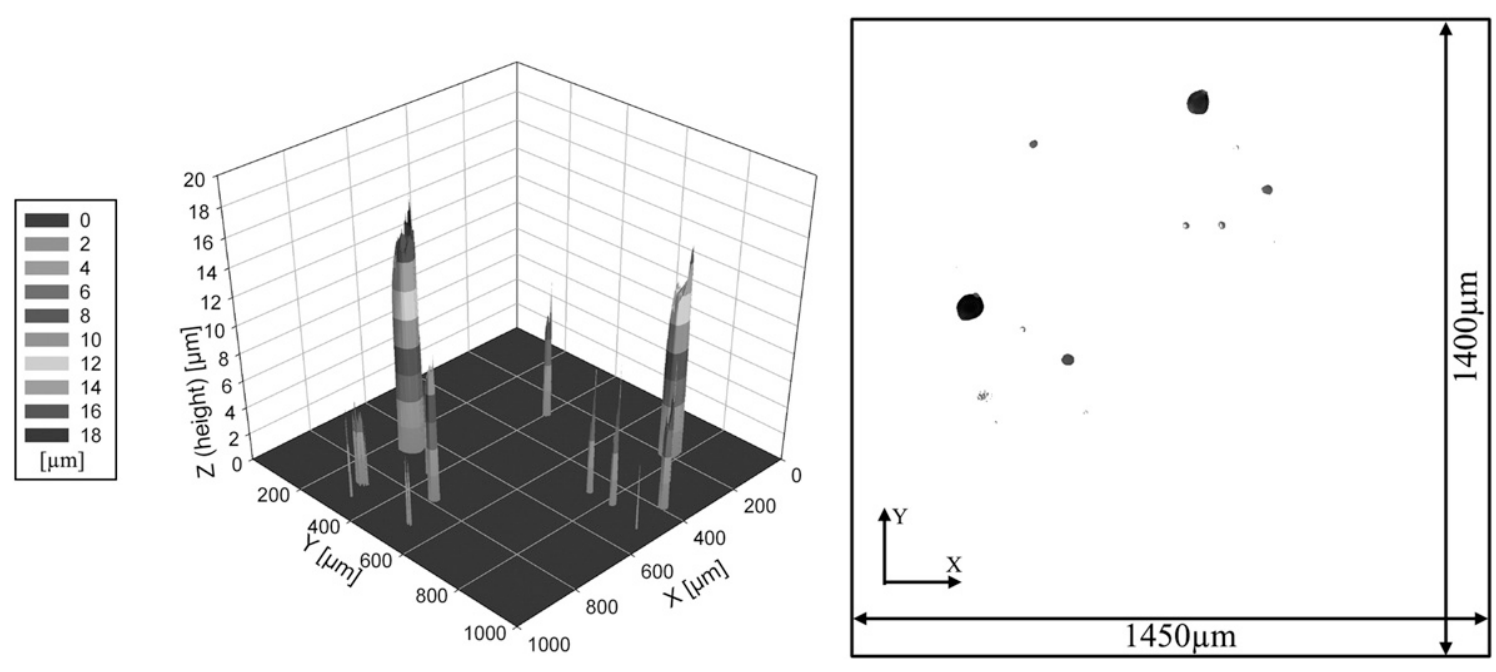

Fig. 2. Corrected three-dimensional and corresponding lateral distribution of the "100 ng As(V)" sample.

Thus one path through the sample was calculated every $1.5 \mu \mathrm{m}$ along the $Y$-axis as shown at the top (4) of Fig. 1. This was done for the entire $Y$-range of the measuring field of the confocal microscope assuming that the whole sample was illuminated by the incident beam.

Considering not only the lateral but also the vertical dimension of the sample, this set of paths was calculated at different heights ranging from zero to the maximum height of the sample. For the simulation only paths with lengths $L_{\text {inc }}$ larger than zero were taken into account.

During the simulation a fixed number of incident photons with one energy value were sent along each of these paths through the sample. So the decision if a fluorescence photon was produced or not had to be made $N$-times by the algorithm described above, where $N$ is given by:

\section{$N=$ number of energy points $\times$ number of photons} $\times$ number of paths (length $>0$ )

To keep the number of paths in a reasonable frame the influence of different step sizes along the $Z$-axis upon the simulation results was investigated and found to be negligible in the range of $100 \mathrm{~nm}$ to $1000 \mathrm{~nm}$ steps. Therefore the $1000 \mathrm{~nm}$ step size was chosen for all simulations which resulted in much shorter simulation times.

For the samples which have not been measured with the confocal microscope (series 1 ) the parameters have been calculated assuming simple sample geometries. The residue of the droplet on the reflector surface was assumed to be a cylinder with diameter $d$ and height $z$.
With this simple geometry $L_{\text {inc }}$ has been calculated as the mean path of a photon penetrating the cylinder parallel to the reflector's surface:

$L_{\text {inc }}=d / 4 \times \pi$

For the calculation of the diameter $d$ the unknown parameters $B$ ("corrected density") and sample height $z$ have been estimated from the data of samples with similar mass of series 2 (i.e. the " $9 \mathrm{~g} \mathrm{As}(\mathrm{V})$ " and the "100 ng As(V)" samples):

$m_{\mathrm{S}} / B=V_{\mathrm{c}}=(d / 2)^{2} \times \pi \times z$

$V_{\mathrm{c}}$ : the volume of the cylinder

$m_{\mathrm{S}}$ : Arsenic mass

Even though these parameters $(B, z)$ have been determined for different samples the simulations showed very good consistence with the measurements (see Section 3).

In the following a short summary of the Monte-Carlo simulation parameters is given:

General assumptions:

- no beam divergence

- beam parallel to reflector-surface

- beam illuminates whole sample
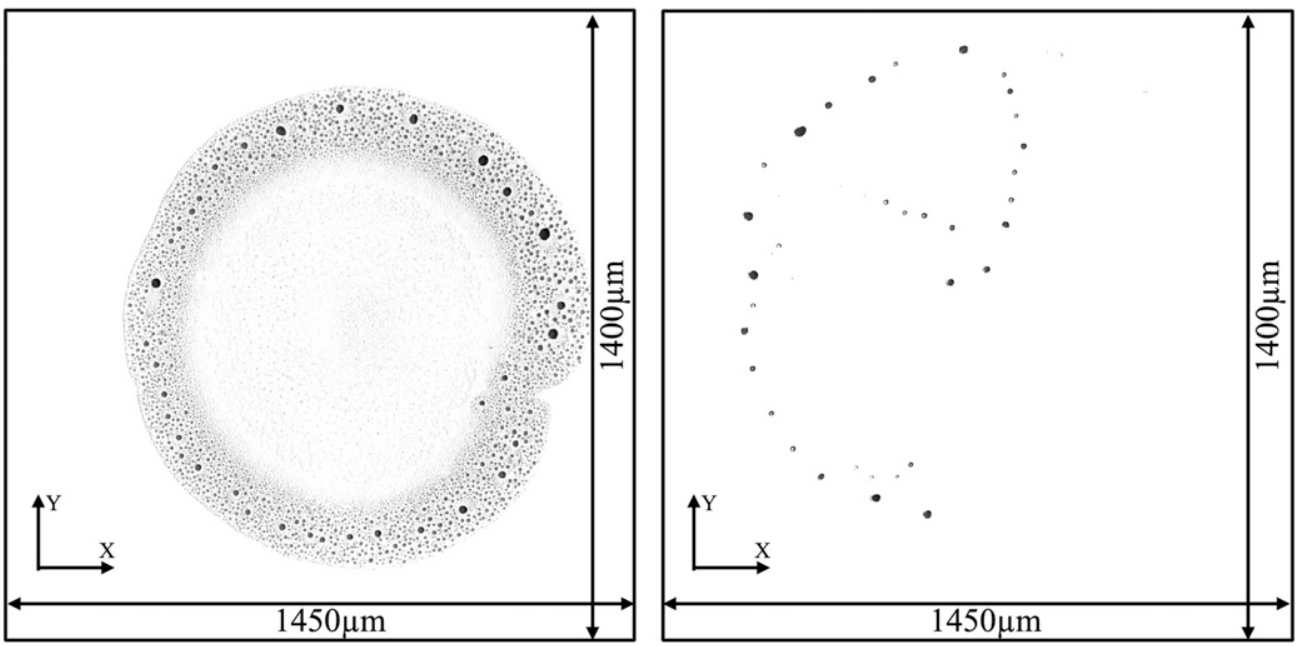

Fig. 3. Corrected distribution of the "500 ng As(V)" (left) and "20 ng As(V)" (right) samples. 

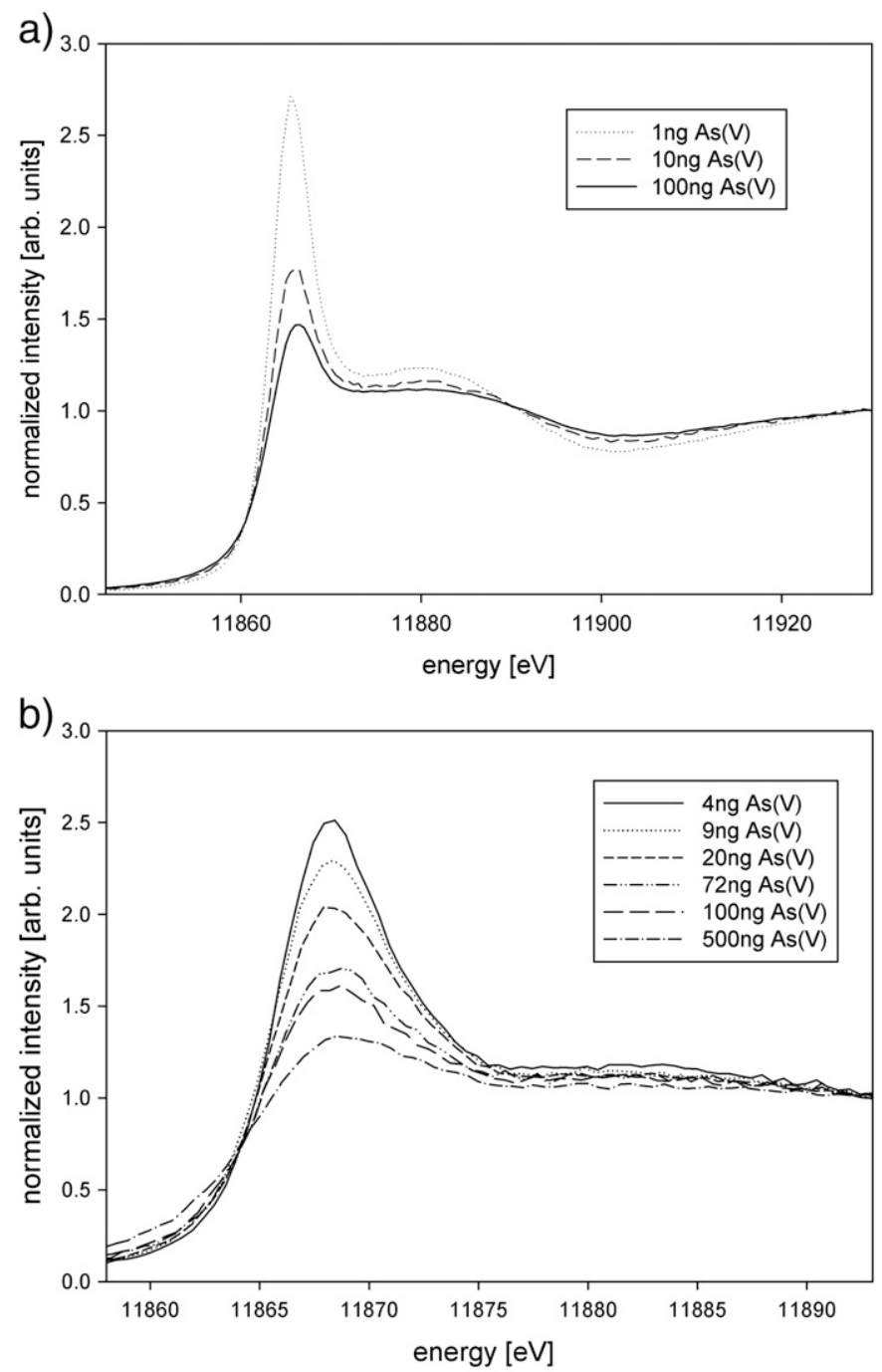

Fig. 4. XANES scans of a) sample series 1 (1, 10, $100 \mathrm{ng}$ of $\mathrm{As}(\mathrm{V})$ on Plexiglas reflectors) and b) sample series 2 (4-500 ng of $\mathrm{As}(\mathrm{V})$ on quartz reflectors) showing the damping of the oscillations.

Parameters for samples of series 1 (no confocal microscope measurements performed):

- simple sample geometry (cylinder, diameter $d$, height $z$ )

- 100,000 photons per simulation

Parameters for samples of series 2 (analyzed with confocal microscope):

- $1000 \mathrm{~nm}$ step size in Z-direction (height) for calculations of paths through sample

- 1000 photons per path through sample

\section{Results and discussion}

\subsection{Results of the measurements with the confocal microscope}

Confocal microscopy images were collected for all the samples of series 2. Fig. 2 reports the images related to sample "100 ng As(V)".

The left part of the figure shows a three-dimensional representation of data gained from the investigation of the sample "100 ng As(V)" of series 2 with the confocal microscope. The scale of the Z-axis (height) is enlarged for clarity. The right part of the figure displays the top view of the whole measuring field $(\sim 1450 \mu \mathrm{m} \times 1400 \mu \mathrm{m})$.

For comparison Fig. 3 shows the data obtained for the samples containing $500 \mathrm{ng}$ (left) and $20 \mathrm{ng}$ (right) $\mathrm{As}(\mathrm{V})$. The datasets presented in both figures were corrected using the procedures described in Section 2.3 .

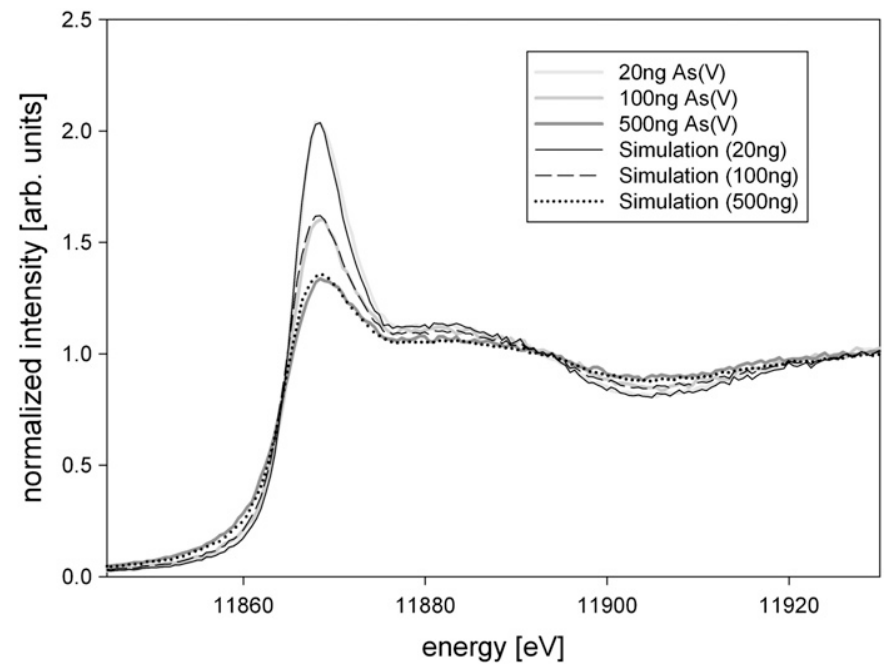

Fig. 5. Measurements and simulations for three samples of series 2 .

\subsection{TXRF-XANES measurements}

Fig. 4 shows the results of the XANES measurements utilizing TXRF geometry which have been performed with sample series 1 (a) and series 2 (b). It can be clearly seen, that the damping of the white line and post-edge oscillations can be correlated to the mass of arsenic. With the energy resolution used for the measurements an energy shift was observed for the $500 \mathrm{ng}$ and the $100 \mathrm{ng}$ samples (about $1.5 \mathrm{eV}$ and $0.5 \mathrm{eV}$ distance between the maxima of the first derivatives of sample "4 ng" and samples " 500 ng" and " 100 ng" respectively). The scan for the "1 ng As(V)" sample was used as reference scan for the MonteCarlo simulations of series 1 and the scan of sample " $4 \mathrm{ng} \mathrm{As}(\mathrm{V})$ " for the simulations of series 2 .

\subsection{Results of the Monte-Carlo simulations}

Fig. 5 shows a comparison of three simulations and their corresponding measured scans. The simulations show good agreement with the measured scans. The damping and broadening of the white line as well as the damping of oscillations at higher energies could be reproduced sufficiently well.

For the simulation of the XANES scans related to the samples of series 1 which have not been investigated with the confocal microscope the parameters $B$ ("corrected density") and $z$ (cylinder

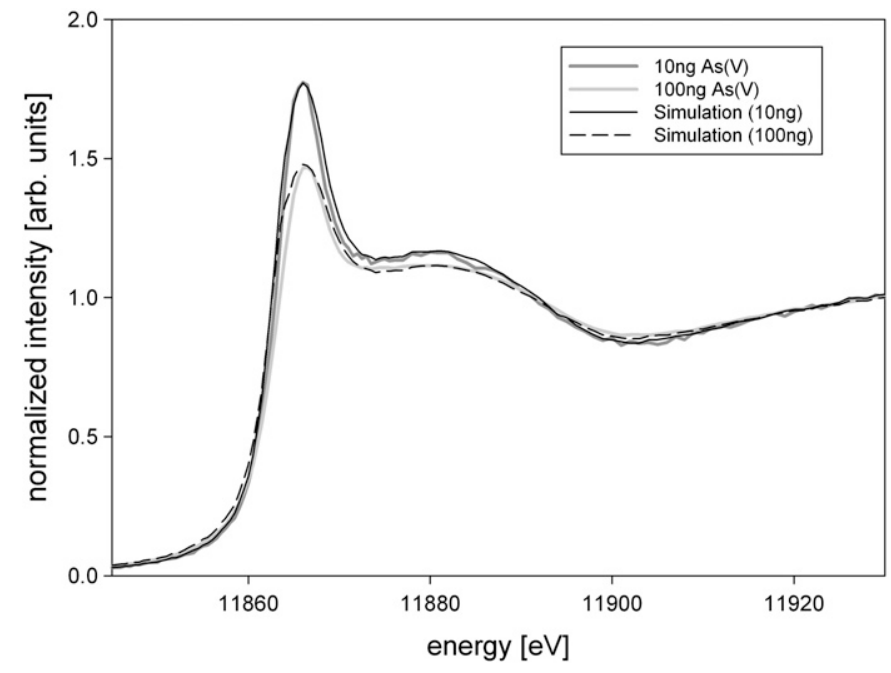

Fig. 6. Measurements and simulations for series 1. 
Table 1

Summary of simulation parameters

\begin{tabular}{|c|c|c|c|c|}
\hline $\begin{array}{l}\text { Simulation for } \\
\text { sample (series 2) }\end{array}$ & $\begin{array}{l}\text { Maximum height } \\
z[\mu \mathrm{m}]\end{array}$ & $\begin{array}{l}\text { "Corrected density" } \\
B\left[\mathrm{~g} / \mathrm{cm}^{3}\right]\end{array}$ & & $\mathrm{Chi}^{2 \mathrm{a}}$ \\
\hline $9 \mathrm{ng} \mathrm{As}(\mathrm{V})$ & 3.7 & 1.22 & & 0.1724 \\
\hline $20 \mathrm{ng} \mathrm{As}(\mathrm{V})$ & 7.3 & 1.24 & & 0.1062 \\
\hline 72 ng As(V) & 11.9 & 0.96 & & 0.2831 \\
\hline 100 ng As(V) & 18.0 & 1.25 & & 0.2126 \\
\hline 500 ng As(V) & 9.7 & 1.34 & & 0.1986 \\
\hline $\begin{array}{l}\text { Simulation for } \\
\text { sample (series } 1 \text { ) }\end{array}$ & $\begin{array}{l}\text { Height of cylinder } \\
z[\mu \mathrm{m}]\end{array}$ & $\begin{array}{l}\text { "Corrected density" } \\
B\left[\mathrm{~g} / \mathrm{cm}^{3}\right]\end{array}$ & $\begin{array}{l}\varnothing \text { of cylinder } \\
d[\mu \mathrm{m}]\end{array}$ & \\
\hline 10 ng $\operatorname{As}(\mathrm{V})$ & 4.0 & 1.22 & 51.1 & 0.1899 \\
\hline $100 \mathrm{ng} \mathrm{As}(\mathrm{V})$ & 15.0 & 1.25 & 82.4 & 0.4839 \\
\hline
\end{tabular}

a $\mathrm{Chi}^{2}$ of the simulation was calculated for the energy range $11,800 \mathrm{eV}$ to $12,000 \mathrm{eV}$.

height) had to be estimated as described in Section 2.4. These parameters defined the dimensions of the cylinder which was the simple approximation of the samples' shape for the calculations. Even though the values of these parameters have been roughly estimated from the data of different samples (series 2) the simulations showed good agreement with the measurements (Fig. 6, Table 1).

Table 1 shows the key parameters of the best simulations for all samples. To evaluate the quality of the simulations the goodness-of-fit parameter chi square was calculated for each simulation: $\mathrm{chi}^{2}=\mathrm{Sum}$ ([simulated value $\left(E_{i}\right)$ - measured value $\left.\left(E_{i}\right)\right]^{2} /$ measured value $\left(E_{i}\right)$ ).

For the calculation of chi square only the XANES region was considered as the interest focuses on the oscillations in this energy range $(11,800-12,000 \mathrm{eV})$.

The values of $B$ for series 2 have been calculated from the data obtained from the measurements with the confocal microscope (i.e. the volume of the samples) and the known mass of the samples. The determined values are small in comparison with the tabulated value of the elemental density of arsenic $\left(5.73 \mathrm{~g} / \mathrm{cm}^{3}\right)$ however they show a surprising consistency. With the exception of the $500 \mathrm{ng}$ sample the maximum heights of the samples show an exponential trend. A reason for the smaller height of the $500 \mathrm{ng}$ sample could be a different drying of the droplet. In Fig. 3 it can be seen that the $500 \mathrm{ng}$ sample has a ring like shape with many small residues inside the ring structure. The $100 \mathrm{ng}$ sample shown in Fig. 2 consists of fewer but larger and higher residues. Despite the differences of these two samples shapes the factor $B$ for both (calculated from their masses and volumes) is very similar. This consistence of $B$ for the samples of series 2 was the reason to hold this parameter constant for the simulations done for series 1 . The height of the cylinder $z$ was also estimated from the values gained for sample series 2 . It was then slightly varied to optimize the simulation with respect to the value of chi square.

\section{Conclusions and outlook}

The topic of the presented work was the influence of selfabsorption effects on TXRF-XANES measurements. The effect of the sample shape as well as the one of different concentrated droplet samples was studied by comparison of calculations and measurements. XANES measurements of two sample series each with different total amounts of mass deposited on quartz and Plexiglas reflectors were carried out under grazing incidence conditions. The results showed a linear correlation of the damping of the oscillations of the scans with the total mass of the samples. It was assumed that this attenuation originates from self-absorption effects caused by the extreme grazing incidence geometry. To verify this hypothesis a simple Monte-Carlo algorithm was developed to simulate these effects. The data about the geometry of the samples required for the simulations was obtained by measurements with a confocal microscope. The simulations performed with this data showed good agreement with the measurements confirming the influence of sample mass and geometry on the damping of the oscillations. On the basis of the data obtained by the measurements with the confocal microscope samples with unknown shape have also been simulated. The results showed good correlation with the measurements as well. The key parameters of this study were the "corrected density" and the length of incidence beam in the sample. It seemed that the shape of the sample is of less importance than the "corrected density" which showed good agreement for all samples. However, the expected differences in the shape of dried residues on Plexiglas and Quartz reflectors due to different surface characteristics will be the topic of further investigations. Another important point related to this topic is the influence of the sample preparation. The deposition of the aqueous sample and the drying process seem to be crucial for quantification in TXRF (especially) without using an internal standard. The best approach to overcome absorption problems seems to be an array of small spots produced with picoliter-pipettes or inkjet printers. This topic is currently under investigation by our group.

The presented results showed that performing an Extended X-ray Absorption Fine Structure (EXAFS) analysis under grazing incidence conditions for higher concentrated samples is very difficult. The damping of the oscillations would make a study of the EXAFS signal almost impossible. A direct correction of the measured scan is not possible because of the loss of information that the phenomenon brings about. For dilute samples on the other hand the measurement time has to be increased drastically to get reasonable counting statistics.

With TXRF acquisition for XANES used as a fingerprint method the investigated self-absorption effect is not dramatic. The energy position of the absorption edge is slightly affected for very high concentrations. This effect does not hinder quantitative evaluations, especially if analysis is carried out by fit of the XANES spectra with analytical functions. However, for a quantitative analysis performed by fitting of scans of unknown samples with those of known reference samples (linear combination method) it would be desirable to have undamped references. Therefore a compromise between counting statistics, measurement time and absorption effects has to be found for the measurement of standard samples.

The presented work proposes a rather simple way to study a priori the absorption effects that will show up in TXRF-XANES and allow the scientist to prepare the sample according to needs (measuring time, acceptable self-absorption for the actual experiment). Moreover the method could be extended to allow the a posteriori correction for the self-absorption of higher concentration standards.

\section{Acknowledgements}

The authors thank Lorenzo Lunelli for the help in processing the raw confocal microscopy images.

This work was supported by the Austrian Science Fund (FWF), project number P18299 and the European Commission, project number II-20042060.

\section{References}

[1] P. Wobrauschek, Total reflection X-ray fluorescence analysis - a review, X-Ray Spectrom. 36 (2007) 289-300.

[2] R. Klockenkamper, Total Reflection X-ray Fluorescence Analysis, Wiley-Interscience, New York, 1997.

[3] C. Streli, G. Pepponi, P. Wobrauschek, C. Jokubonis, G. Falkenberg, G. Zaray, A new SR-TXRF vacuum chamber for ultra-trace analysis at HASYLAB, Beamline L, X-Ray Spectrom. 34 (2005) 451-455.

[4] C. Streli, G. Pepponi, P. Wobrauschek, C. Jokubonis, G. Falkenberg, G. Zaray, J. Broekaert, U. Fittschen, B. Peschel, Recent results of synchrotron radiation induced total reflection X-ray fluorescence analysis at HASYLAB, Beamline L, Spectrochim. Acta Part B 61 (2006) 1129-1134.

[5] A. Singh, K. Baur, S. Brennan, T. Homma, N. Kubo, P. Pianetta, X-ray absorption spectroscopy on copper trace impurities on silicon wafers, MRS Proceedings, vol. 716, 2002. 
[6] G. Falkenberg, G. Pepponi, C. Streli, P. Wobrauschek, Comparison of conventional and total reflection excitation geometry for fluorescence X-ray absorption spectroscopy on droplet samples, Spectrochim. Acta Part B 58 (2003) 2239-2244.

[7] F. Meirer, G. Pepponi, C. Streli, P. Wobrauschek, V.G. Mihucz, G. Záray, V. Czech, J.A.C. Broekaert, U.E.A. Fittschen, G. Falkenberg, Application of synchrotronradiation-induced TXRF-XANES for arsenic speciation in cucumber (Cucumis sativus L.) xylem sap, X-Ray Spectrom. 36 (2007) 408-412.

[8] G. Pepponi, B. Beckhoff, T. Ehmann, G. Ulm, C. Streli, L. Fabry, S. Pahlke, P. Wobrauschek, Analysis of organic contaminants on Si wafers with TXRF-NEXAFS, Spectrochim. Acta Part B 58 (2003) 2245-2253.

[9] J. Osán, B. Török, S. Török, K.W. Jones, Study of chemical state of toxic metals during the life cycle of fly ash using X-ray absorption near-edge structure, X-Ray Spectrom. 26 (1997) 37-44.

[10] F. Goodarzi, F.E. Huggins, Speciation of arsenic in feed coals and their ash byproducts from Canadian power plants burning sub-bituminous and bituminous coals, Energy Fuels 19 (2005) 905-915.

[11] L. Tröger, D. Arvanitis, K. Baberschke, H. Michaelis, U. Grimm, E. Zschech, Full correction of the self-absorption in soft-fluorescence extended X-ray-absorption fine structure, Phys. Rev. B 46 (1992) 3283.

[12] P. Pfalzer, J.P. Urbach, M. Klemm, S. Horn, M.L. denBoer, A.I. Frenkel, J.P. Kirkland, Elimination of self-absorption in fluorescence hard-X-ray absorption spectra, Phys. Rev. B 60 (1999) 9335
[13] www.radiantdetectors.com/vortex.html, The SII Nanotechnology Incorporated Website, 2007.

[14] G. Falkenberg, Characterization of a radiant vortex silicon multi-cathode X-ray spectrometer for (total reflection) X-ray fluorescence applications, Hasylab Internal Report 2004, 2005.

[15] http://cars9.uchicago.edu/ifeffit/, The IFEFFIT homepage. 2007.

[16] M. Newville, IFEFFIT: interactive XAFS analysis and FEFF fitting, Journal of Synchrotron Radiation 8 (2001) 322-324.

[17] B. Ravel, M. Newville, ZATHENA, ARTEMIS, HEPHAESTUS: data analysis for X-ray absorption spectroscopy using IFEFFIT, J. Synchrotron Radiat. 12 (2005) 537-541.

[18] http://www.nanofocus.info, The Nanofocus AG homepage, 2007.

[19] www.imagemet.com, The Image Metrology A/S homepage, 2007.

[20] B.L. Henke, E.M. Gullikson, J.C. Davis, X-ray interactions: photoabsorption, scattering, transmission, and reflection at $E=50-30,000 \mathrm{eV}, Z=1-92$, Atomic Data and Nuclear Data Tables, Vol/Issue: 54:2, DOE Project, 1993, pp. 181-342.

[21] H. Ebel, R. Svagera, M.F. Ebel, A. Shaltout, J.H. Hubbell, Numerical description of photoelectric absorption coefficients for fundamental parameter programs, X-Ray Spectrom. 32 (2003) 442-451.

[22] M. Makoto, K. Yoshiharu, Twisted GFSR generators II, ACM Trans. Model. Comput. Simul. 4 (1994) 254-266. 